\title{
Force Feedback Control based on VGSTA for Single Track Riding Simulator
}

\author{
Lamri Nehaoua and Hichem Arioui and Leonid Fridman
}

\begin{abstract}
A direct application of the variable-gain supertwisting algorithm (VGSTA) is implemented for torque feedback on a handlebar of a riding simulator. This control strategy aims to compensate perturbations changing with the system states. Thanks to the good tracking performance and robustness/insensitiveness of such a control method, a precise estimation of the rider's torque applied on the riding simulator handlebar is possible. A first-order sliding-mode observer with stabilization is designed for the estimation of the unknown input rider action. Experimental implementation and analysis are provided to point-out the effectiveness of the proposed approach.
\end{abstract}

\section{INTRODUCTION}

In the past, motorcycles were seen as distraction tool than transportation one. But during the last decade and under stress of daily life constraints, riders' attitudes have evolved. Its squeeze ability and energy consumption have made her an indispensable means of transportation, especially in large cities, despite the associated risks.

Nowadays, the driving safety has become the primary concern of authorities. Thanks to high advances of mechatronic devices, driving simulator are now widely used for risk and driving behavior studies. A driving simulator is composed of various sub-systems including the mechanical platform, visual environment and associated softwares [1], [2]. Even that driving is mainly a visual task, the multiplication of sensory cues allows to enhance the simulation fidelity. Among these cues, torque feedback on simulator handlebar/steeringwheel is one of the most important features of a successful driver immersion. Nevertheless, in car driving simulator, torque feedback is intended to supply the driver with a good information on road friction and hence, a small restitution of the real induced torque is sufficient.

However, riding a two-wheeled vehicle is more complex deal than driving a car one. In addition to the road path following, the rider must ensures the vehicle stabilization. For example, in a turn maneuver, handy counter-steering is required beyond a given speed. This action is achieved mainly by applying an adequate torque on the motorcycle's handlebar in order to compensate tire/road efforts, that spread along the steering mechanism, and thus tilt the bike with a desired roll angle to hold its equilibrium. This example, among others, highlights that two-wheeled vehicles are mainly driven by the handlebar applied torque rather than

This work was not supported by any organization

Lamri Nehaoua and Hichem Arioui are with IBISC, University of Evry, 91020, France $\{$ nehaoua, arioui\}@ibisc.univ-evry. fr

Leonid Fridman is with the Department of Control and Robotics Engineering, UNAM, Mexico Ifridman@servidor. unam.mx the wheel steer angle. Consequently, in a motorcycle riding simulator, a full-scale torque feedback must be realized.

Most of the torque control feedback is done by computing at each sample time the resulting self-alignment tire-road effort and next, a PID control is implemented for the DC motor current tracking. In [3], torque feedback is designed by modeling the efforts flux exchange, between tire/road contact and the vehicle driver, as a two-part network haptic interaction. In this paper, the torque feedback is implemented as a model-reference following approach, in which, handlebar steer angle reference is computed. Next, a robust perturbation rejection control for the reference tracking is designed. This control approach is more efficient since it avoids the recompilation of the resulted torque, requiring rate and acceleration information, and also avoids algebraic loops which may cause numerical instabilities.

In this field, Sliding Mode Control (SMC) is a well known approach for rejecting matched perturbations (uncertainties/disturbances) [4]. This control approach has greatly matured recently to overcome associated disadvantages such as chattering and to be adapted to the real-time implementation constraints. The High-Order Sliding-Mode (HOSM) methods, like Super-Twisting Algorithm (STA) are widely used [5]. However, the homogeneous nature of the standard STA does not allow to compensate perturbations growing simultaneously with the state variables. That is why it is very important to design non homogeneous extension of the standard STA with variable gains (VGSTA) [6].

This paper is organized as follows: next section is dedicated to the problem statement. In section 3, a sliding-mode observer is used for the estimation of the rider torque exerted on the simulator's handlebar. Section 4 deals with a direct application of the VGSTA to implement a handlebar torque feedback. Experimentation and conclusion wrap up the paper.

\section{Problem Statement}

Actuate the simulator's mechanical platform amounts to setting its states motion in accordance with the user's desired actions. As shown in figure (1), rider's actions are the main inputs to the simulator software which includes, among others, the vehicle model. This model aims to compute the dynamics of a virtual motorcycle where the resulting states will be serve as a basis to generate reference trajectories.

Since the motorcycle is mainly controlled by the applied rider's steering torque and due to the absence of a torque sensor, a precise estimation of the rider's torque applied on the simulator's handlebar is necessary. Moreover, a good torque feedback on the simulator's handlebar is mandatory. 
To achieve this goal, the simulator's handlebar is attached to a DC motor via a double pulley-belt system. An optical encoder is mounted on the motor axis for the angle measurement [7]. The use of the pulley-belt system is a suitable solution to enhance the resolution of the position measure and for the multiplication of the maximum allowable motor torque. The resulting system constitutes the new interface system between the simulator's rider and the virtual motorcycle.

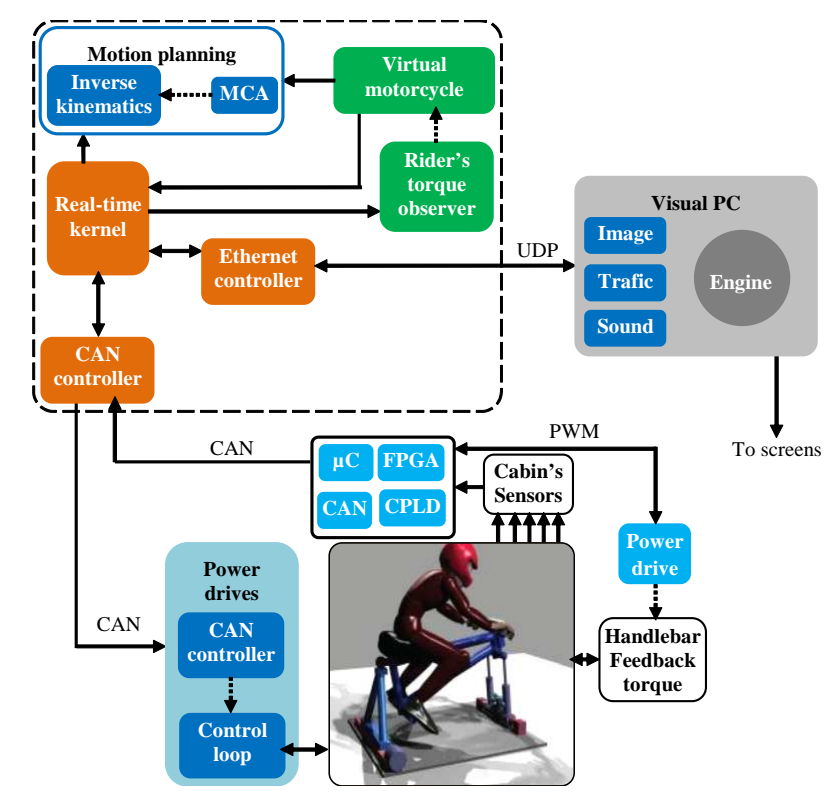

Fig. 1. Overall motorcycle simulator mechatronic architecture

From this discussion, it arises that our aims is twofold:

- given the handlebar position and motor current information, design an observer to estimate the the unknown torque input applied by the rider on the simulator's handlebar,

- design a handlebar full-scale feedback torque.

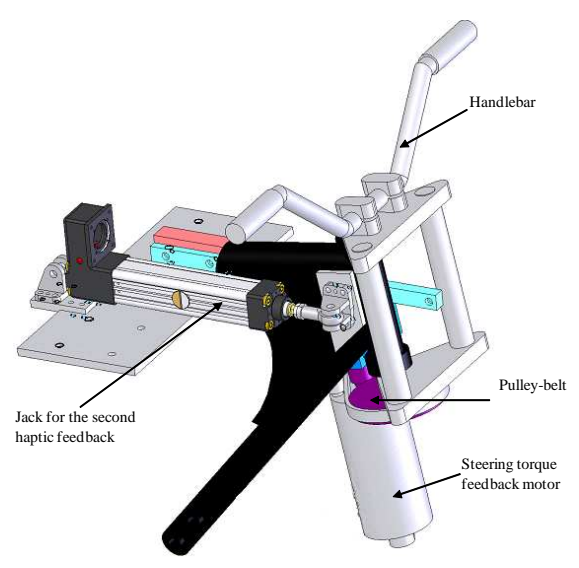

Fig. 2. Haptic device for torque feedback

\section{SLIDING MODE OBSERVER DESIGN FOR THE RIDER'S TORQUE ESTIMATION}

In this section, we focus on the applied rider's torque estimation which is considered as an unknown input. For this, two sliding mode observers (SMO) will be designed in view of comparison. The first SMO is that of WalcottZak (WZO) [8], which is an extension of the well known $1^{\text {st }}$ order SMO [9]. The second one is based on the supertwisting algorithm (STAO) [10].

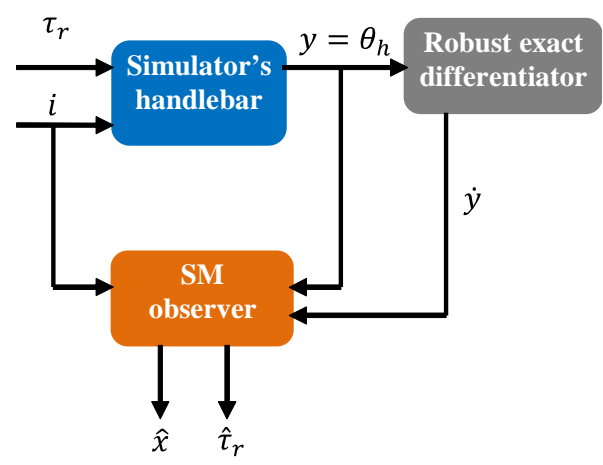

Fig. 3. Unknown input sliding observer diagram

The state-space representation of the new handlebar interface is derived given the mechanical equation of the handlebar/motor assembly as following:

$$
\begin{aligned}
& \dot{x}=\mathcal{A} x+\mathcal{B} u+\mathcal{D} \zeta+\tau_{s p} \\
& y=\theta_{h}=\mathcal{C} x
\end{aligned}
$$

where, $x=\left[\dot{\theta}_{h}, \theta_{h}\right]^{T}$ is the state vector including handlebar position and position rate, $u=k_{t} N i$ is the control input, $\zeta=\tau_{r}$ is the rider's torque which is a bounded Lebesgue measurable unknown input $\|\zeta\| \leq \rho, \rho \succ 0$. $\tau_{s p}=$ $-\beta_{s} \operatorname{sign}\left(\dot{\theta}_{h}\right)$ is considered as a known external bounded perturbation [11] and $\mathcal{D}, \mathcal{C}$ are respectively of full column and row rank.

For system (1), it is easy to check that the observer matching condition is not full-filed since $\operatorname{rank}(\mathcal{D}) \neq \operatorname{rank}(\mathcal{C D})$. Nevertheless, according to [12], the WZO can be applied by generating the estimation of the handlebar position rate (Fig.3). Hence, the new output matrix is computed according to the relative degree of the system with respect to each output. In our case, $\mathcal{C}_{a}$ is the $2 \times 2$ identity matrix. Then, we can make use of the following WZO equation :

$$
\dot{\hat{x}}=\mathcal{A} \hat{x}+\mathcal{B} u+\mathcal{L}\left(y_{a}-\hat{y}_{a}\right)-\mathcal{D} E\left(\hat{y}_{a}, y_{a}, \eta\right)
$$

where $y_{a}=[y, \dot{y}]$ and the generated additional output $\dot{y}$ is obtained by a robust super-twisting exact differentiator [13]. $\hat{y}$ is the output estimate. $E(\hat{y}, y, \eta)$ is the discontinuous injection term which depends on the output estimation error. The term $\mathcal{L}(y-\hat{y})$ is intended for stabilization where the gain matrix $\mathcal{L}$ can be freely assigned $((\mathcal{A}, \mathcal{C})$ is observable). After a finite time convergence (exact convergence if the measured 
output $y$ is noise free), the unknown input is estimated by (proof in [8]):

$$
\zeta=-E\left(\hat{y}_{a}, y_{a}, \eta\right)
$$

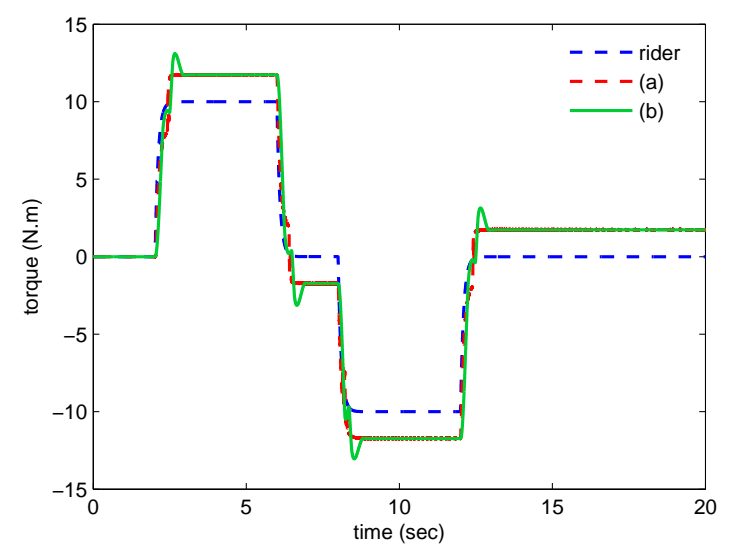

Fig. 4. Example of an estimated rider's torque using simulation test, (a) using WZO, (b) using STAO
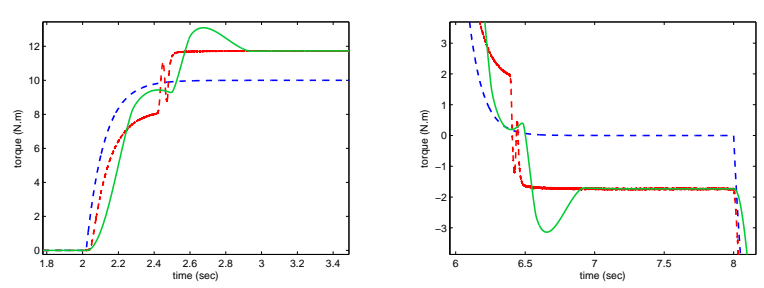

Fig. 5. Zoom of figure 4. At right zoom in [1.8s-3.5s], at left zoom in [6s-8.5s]

Unfortunately, the realization of the WZO requires filtration due to the discontinuous term and the time discretization effect. Recently, thanks to STA, the developed hierarchical observers (STAO) avoid the filtration and provide asymptotic accuracy of the derivative estimation at each single realization step [9], [10]. Nevertheless, STAO require knowledges about the unknown input successive derivatives. According to [10], system (1) satisfy the following assumptions:

(a) system (1) has a relative degree $r=n=2$ w.r.t the unknown input, hence, it is strongly observable,

(b) $\zeta(t)$ is a bounded Lebesgue measurable unknown input $\|\zeta\| \leq \rho, \rho \succ 0$

(c) $\zeta(t)$ is bounded with successive derivatives up to the order $k$ bounded by the same constant as $\left\|\zeta^{(k+1)}(t)\right\| \leq$ $\rho^{\prime}, \rho^{\prime} \succ 0$ (herein $k=1$ ).

Next, the observer is implemented by the following expression:

$$
\begin{aligned}
& \dot{z}=\mathcal{A} z+\mathcal{B} u+\mathcal{L}(y-\mathcal{C} z) \\
& \hat{x}=z+\mathcal{K} v \\
& \dot{v}=\Lambda(y-\mathcal{C} z, v)
\end{aligned}
$$

where, vector $v$ and the discontinuous term $\Lambda(y-\mathcal{C} z, v)$ are chosen differently depending on what the system is strong observable or strong detectable (see [10] for equations and proofs).

Figure (4) illustrates an example of a simulated \pm 10 (N.m) steering torque and its estimation by applying the unknown input WZO and STAO (equations (2) and (3)). As shown in this figure, both observers provide time finite convergence and exact unknown input estimation but STAO are more suitable since they do not require filtering and they are numerically more stable. We note that the gap, between the reference and estimated torques, is mainly due to the dry friction component. Indeed, torque plot gives the absolute torque $\tau_{r}+\beta_{s} \operatorname{sign}\left(\dot{\theta}_{h}\right)$ and to recover the rider's torque, one must compensate for the dry friction. This compensation will be covered in the following section.

\section{TORQUE FEEDBACK CONTROL}

As aforementioned, the rider torque is considered as the main input to the virtual motorcycle dynamic model (Figure 6). On simulator, the rider should be able to drive a virtual motorcycle as close as a real drive situation. For this end, it is crucial to feedback torque information at full-scale.

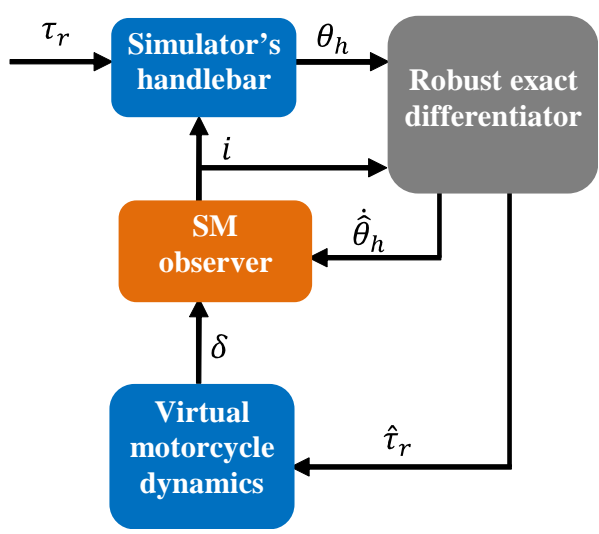

Fig. 6. Diagram for interfacing the virtual motorcycle and the simulator's handlebar

Consider the present handlebar system state-space equation (1). Due to its form, this equation can be written as:

$$
\dot{x}=\mathcal{A} x+\mathcal{B}(u+f(x, t))
$$

where $x, u$ are same signals in equation (1) and $f(x, t)=$ $\tau_{r}-\beta_{s} \operatorname{sign}\left(\dot{\theta}_{h}\right)$ is a disturbance including the continuous riding torque $\tau_{r}$ and a bounded piecewise smooth perturbation related to the dry friction.

When the perturbation $f(x, t)$ is bounded by a known function $\rho(x)$ such that $|f(x, t)| \leq \rho(x)$, the first-order Sliding Mode Controller (SMC) $\nu=-\rho(x) \operatorname{sign}(\sigma)$ can be used even for a non differentiable perturbations at the expense of a discontinuous control (chattering). The system under control is of relative degree 2, thus, we are using VGSTA to avoid the chattering otherwise we can use twisting algorithm 
(STA) and do not use the switching surface. The SuperTwisting Algorithm (STA) were designed as an absolutely continuous control law allowing to compensate Lipschitz unbounded perturbations but with a bounded time derivative [14]. However, we need VGSTA because the uncertainties are bounded with known function.

In the reminder of this section, a direct application of the VGSTA is used for the control purpose of this torque feedback. More details and proofs are available in [6].

For the current system, $\mathcal{A}$ and $\mathcal{B}$ have the following forms:

$$
\mathcal{A}=\left[\begin{array}{cc}
a_{11} & 0 \\
1 & 0
\end{array}\right] \quad \mathcal{B}=\left[\begin{array}{l}
b \\
0
\end{array}\right]
$$

For this system, the following assumption are true:

1) $\mathcal{B}$ is full column rank, i.e., $\operatorname{rank}(\mathcal{B})=2$,

2) The pair $(\mathcal{A}, \mathcal{B})$ is controllable,

3 ) The disturbance $f(x, t)$ and its gradient are bounded.

From assumption (1) and (2), one can find a linear transformation $\mathcal{T}$ for which, the system of equation (4) can be made in the following regular form:

$$
\left[\begin{array}{c}
\dot{\eta} \\
\dot{\xi}
\end{array}\right]=\mathcal{T} \mathcal{A T}^{-1}\left[\begin{array}{l}
\eta \\
\xi
\end{array}\right]+\mathcal{T} \mathcal{B}\left(u+f_{1}(\eta, \xi, t)\right)
$$

Referring to the equation (5), a proper choice of $\mathcal{T}$ as:

$$
\mathcal{T}=\left[\begin{array}{c}
\mathcal{B}^{\perp} \\
\mathcal{B}^{\dagger}
\end{array}\right] \rightarrow \mathcal{T}=\left[\begin{array}{cc}
0 & -b \\
b^{-1} & 0
\end{array}\right]
$$

allows to obtain the new system representation as:

$$
\left\{\begin{array}{l}
\dot{\eta}=-b^{2} \xi \\
\dot{\xi}=a_{11} \xi+u+f_{1}(\eta, \xi, t)
\end{array}\right.
$$

The main idea of sliding mode control is to design the sliding surface variable:

$$
\sigma=\xi-K \eta
$$

such that, when the motion reaches the sliding surface $(\sigma=0)$, the reduced-order model $\left(\dot{\eta}=-b^{2} K \eta\right)$ has the prescribed performance. To compensate for disturbance $f_{1}(\eta, \xi, t)$, an injection term $\nu$ is added to the equivalent control in order to form the global control variable $u$. By using variables $[\eta, \sigma]^{T}$ as state vector, the control signal is expressed by:

$$
u=u_{e q}+\nu=-\left(a_{11}+K b^{2}\right) K \eta-\left(a_{11}+K b^{2}\right) \sigma+\nu
$$

and the system representation (8) can be transformed to the following one :

$$
\left\{\begin{array}{l}
\dot{\eta}=-b^{2} \xi \\
\dot{\sigma}=\nu+f_{1}(\eta, \sigma+K \eta, t)
\end{array}\right.
$$

in which, $\nu$ is taken to be a super-twisting algorithm with variable gain (VGSTA) :

$$
\nu=-k_{1}(t, x) \varphi_{1}(\sigma)-\int_{0}^{t} k_{2}(t, x) \varphi_{2}(\sigma) d t
$$

where:

$$
\begin{aligned}
\varphi_{1}(\sigma) & =\sqrt{|\sigma|} \operatorname{sign}(\sigma)+k_{3} \sigma \\
\varphi_{2}(\sigma) & =\frac{d \varphi_{1}(\sigma)}{d \sigma} \varphi_{1}(\sigma)
\end{aligned}
$$

here, $k_{1}, k_{2}$ are variable gains which make the sliding surface insensitive to perturbations growing with bounds given by known functions. $k_{3}$ allows to deal with perturbations growing linearly in $\sigma$. When $k_{3}=0, k_{1}$ and $k_{2}$ are constant gains, equation (12) recovers the standard supertwisting algorithm.

To define the class of perturbations that VGSTA can compensates, uncertainty/disturbance are divided into two parts:

$$
f_{1}(\eta, \sigma+K \eta, t)=g_{1}(\eta, \sigma, t)+g_{2}(\eta, t)
$$

So, according to assumption (3), VGSTA is insensitive to perturbations $f(x, t)$ satisfying:

$$
\begin{aligned}
\left|g_{1}(\eta, \sigma, t)\right| & \leq \rho_{1}(t, x)\left|\varphi_{1}(\sigma)\right| \\
\left|\frac{d}{d t} g_{2}(\eta, t)\right| & \leq \rho_{2}(t, x)\left|\varphi_{2}(\sigma)\right|
\end{aligned}
$$

where, $\rho_{1}(t, x)$ and $\rho_{2}(t, x)$ are both a positive known continuous functions. Finally, system (11) controlled by the VGSTA algorithm (12) can be rewritten as:

$$
\left\{\begin{array}{l}
\dot{\eta}=-b^{2} \xi \\
\dot{\sigma}=-k_{1}(t, x) \varphi_{1}(\sigma)+g_{1}(\eta, \sigma, t)+z \\
\dot{z}=-k_{2}(t, x) \varphi_{2}(\sigma)+\dot{g}_{2}(\eta, t)
\end{array}\right.
$$

where, for the present problem $g_{1}(\eta, \sigma, t)=0$ and :

$$
g_{2}(\eta, t)=\tau_{r}-\beta_{s} \frac{\dot{\eta}}{\sqrt{\dot{\eta}^{2}+\epsilon^{2}}}
$$

and $\epsilon$ is a design parameter.

\section{Simulation RESUlts AND EXPERIMENTAL tests}

The aim of the present tests is to point-out the effectiveness of the overall architecture of figure (6), including unknown input observer, exact differentiator and the VGSTA control for the torque feedback. We discuss the reconstruction of the applied rider's torque results and the steer angle tracking performance.

Figure (7) illustrates the simulated steering torque versus that estimated, by applying the unknown input observer of equation (3), where we observe a exact convergence in a finite time. This result is achieved thanks to the ability of the VGSTA to compensate the dry friction perturbation in the torque feedback control expressed by the goos steer angle tracking performance (Figure (8)). 


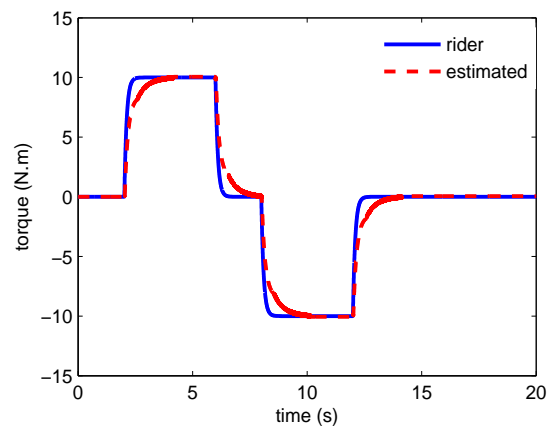

Fig. 7. Estimation of \pm 10 (N.m) unknown input torque

In figures (9-11), we present experiments carried out on the handlebar system of the motorcycle riding simulator. A lane change maneuver with different longitudinal speeds (100 and $40 \mathrm{~km} / \mathrm{h}$ ) were performed.
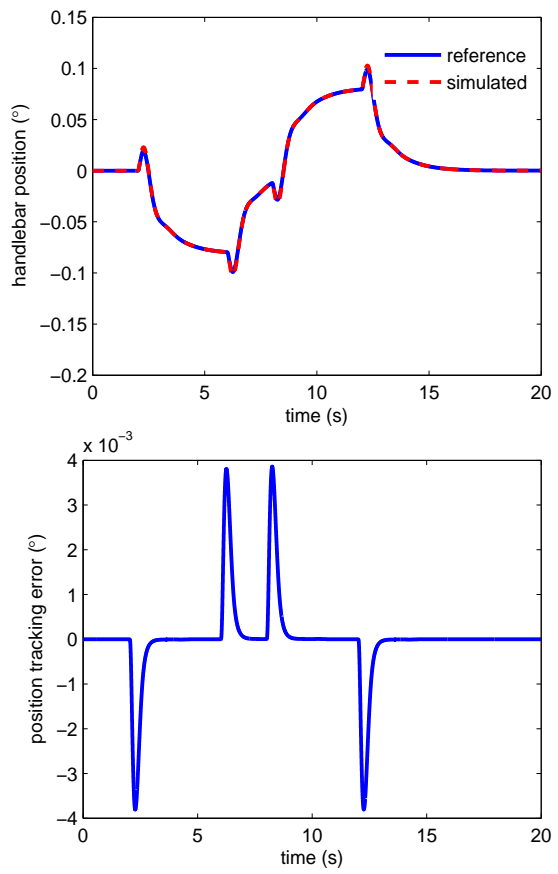

Fig. 8. Steer angle tracking and the associated error
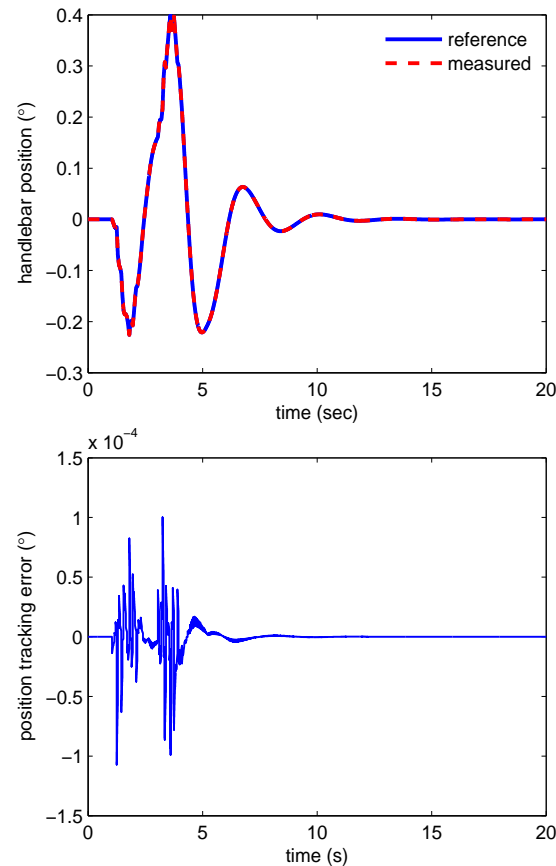

Fig. 9. Steer angle tracking using VGSTA for a double lane change at $100(\mathrm{~km} / \mathrm{h})$
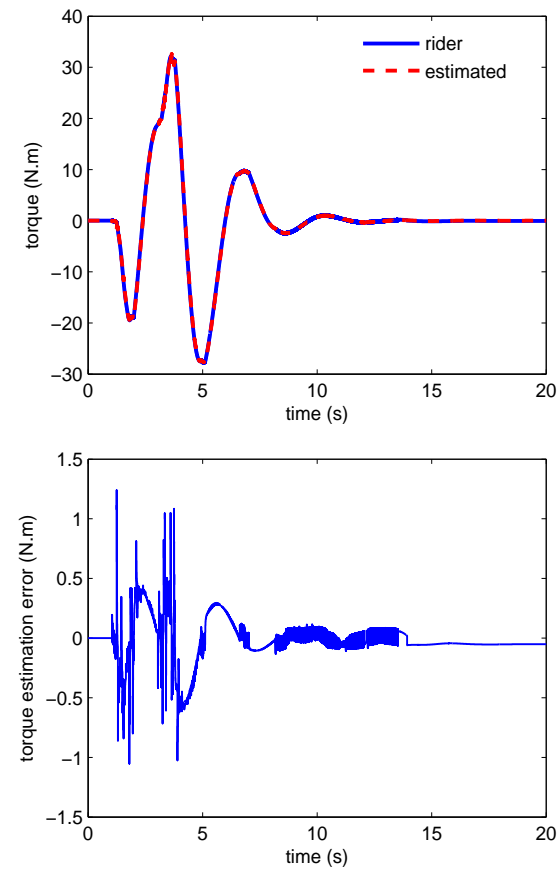

Fig. 10. Rider's torque estimation using the sliding mode observer and differentiator for a double lane change at $100(\mathrm{~km} / \mathrm{h})$ 

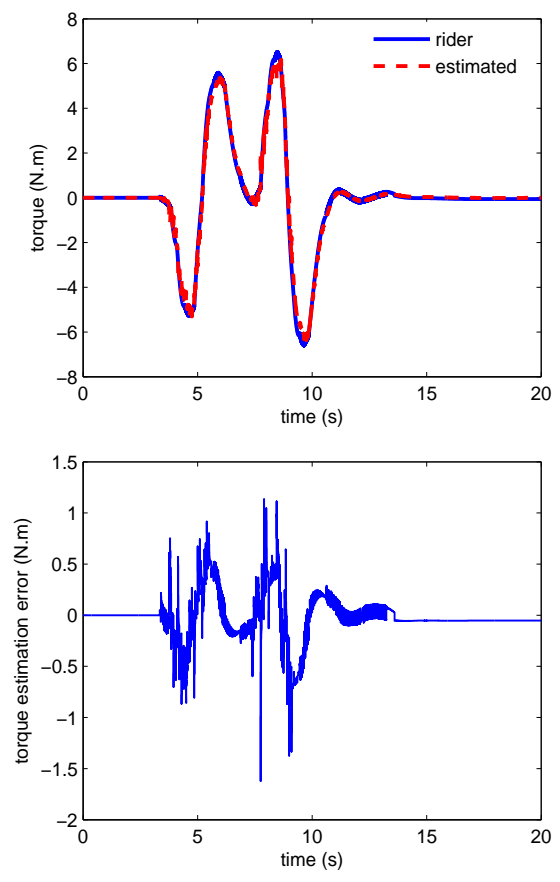

Fig. 11. Rider's torque estimation using the sliding mode observer and differentiator for a double lane change at $40(\mathrm{~km} / \mathrm{h})$

At $100(\mathrm{~km} / \mathrm{h})$, one can notice the good estimation of the driver torque (Figure 9) and the exact tracking of the handlebar steer angle even for very small handlebar position reference (Figure 10).

At $40(\mathrm{~km} / \mathrm{h})$, the quality of riders torque estimation remains intact even the greater impact of the dry friction torque (figure 11). The compensation for this component is highly suitable in this case (at low speed).

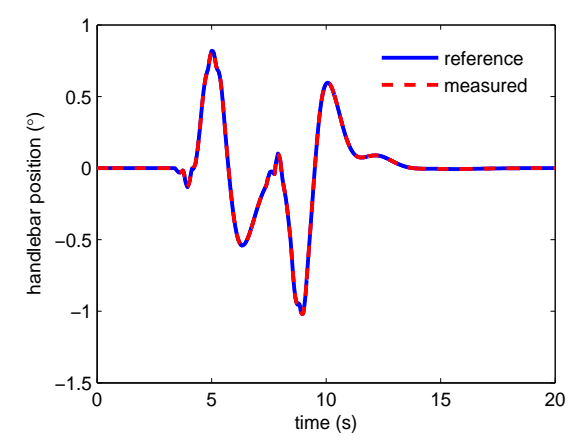

Fig. 12. Steer angle tracking using VGSTA for a double lane change at $40(\mathrm{~km} / \mathrm{h})$

Figure (13) shows a comparison between the VGSTA and an $\mathrm{H}_{\infty}$ control performance. In the $\mathrm{H}_{\infty}$ method, a feedback loop-shaping design procedure is adopted using the normalized left coprime factor [15]. In this figure, the quality of rider's torque estimation using the $\mathrm{H}_{\infty}$ method for the torque feedback control is quite imprecise because of the greater impact of the dry friction torque. This method falls in the compensation of a piecewise friction perturbations.

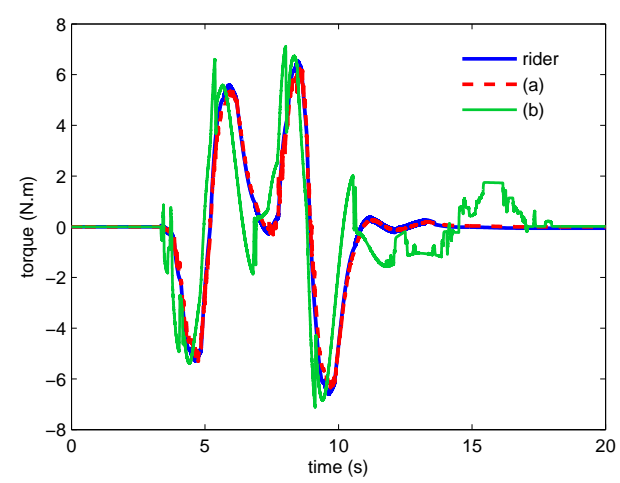

Fig. 13. Comparison between the VGSTA (a) and an $\mathrm{H}_{\infty}$ control (b) performance for a double lane change at $40(\mathrm{~km} / \mathrm{h})$

\section{CONCLUSION}

In this paper we present a real application of a sliding mode unknown input observer for rider's torque estimation. The estimated torque is used to drive a virtual motorcycle dynamics model and hence be able to ride a motorcycle simulator. Since, the handlebar plant does not satisfy the matching observer condition, additional outputs are generated by using a robust exact differentiator.

Next, the handlebar torque feedback is developed. This feedback is designed as a model-reference following on the handlebar steer rather than a motor current control. For this, a direct application of the VGSTA is achieved. This approach is chosen because it generates an absolutely continuous control ensuring chattering reduction and for the exact compensation of (piecewise) Lipschitz continuous perturbation with gradients bounded by known functions. The effectiveness of the overall architecture is simulated and tested. For our current application (driving simulation), obtained results are highly acceptable even at low speed where the dry friction should be compensated.

Future work will deal with the psycho-physical validation of the proposed control method. The present simulator will be tested with several riders in order to acquire their feeling of the torque feedback and thus for various riding scenarios. Other questions which are arising during the implementation of the VGSTA will be investigated such that: if we need to use also variable gain differentiator? What about to put a third order sliding mode through integrator. These questions could be investigated in the next steps.

\section{REFERENCES}

[1] L. Nehaoua, H. Mohellebi, A. Amouri, H. Arioui, S. Espié and A. Kheddar, "Design and control of a small-clearance driving simulator". IEEE Transactions on Vehicular Technology 2008, vol. 57(1), pp 736746.

[2] L. Nehaoua, S. Hima, H. Arioui, N. Séguy and S. Espié, "Design and modeling of a new motorcycle riding simulator". in Proceedings of the 2007 American Control Conference, pp 176-181, New York, USA, 2007.

[3] H. Mohellebi, A. Kheddar and S. Espié, "Adaptive Haptic Feedback Steering Wheel for Driving Simulators", IEEE Transactions on Vehicular Technology 2009, vol. 58(4), pp 1654-1666.

[4] H.K. Khalil, "Nonlinear Systems", Third edition, Prentice Hall, Upsaddle River, NJ; 2002. 
[5] G. Bartolini, A. Ferrara and E. Usai, "Chattering avoidance by second order sliding mode control", IEEE Transactions on Automatic and Control 1998, vol. 43(2), pp 241-246.

[6] A. Dàvila, J.A. Moreno and L. Fridman, "Variable Gains SuperTwisting Algorithm: A Lyapunov Based Design", in Proceedings of the 2010 American Control Conference, pp 241-246, Baltimore, USA.

[7] H. Arioui, L. Nehaoua, S. Hima, N. Séguy and S. Espié, "Mechatronics, design and modeling of a motorcycle riding simulator", IEEE/ASME Transactions on Mechatronics 2010, vol. 15(5), pp 805818.

[8] S. Hui and S.H. Zak, "Observer design for systems with unknown inputs", International Journal of Applied Mathematics and Computer Science 2005, vol. 15(14), pp 431-446.

[9] J.F. Bejarano, L. Fridman, A. Poznyak, "Exact state estimation for linear systems with unknown inputs based on hierarchical supertwisting algorithm",International Journal on Robust and Nonlinear Control 2007, vol. 17(18), pp 1734-1753.

[10] L. Fridman, A. Levant, J. Davila, "Observation of Linear Systems with Unknown Inputs via High-Order Sliding-Mode", International Journal of Systems Science 2007, vol. 38(10), pp 773-791.

[11] J. Davila, L. Fridman and A. Levant, "Second-Order Sliding-Mode Observer for Mechanical Systems", IEEE Transactions on Automatic and Control 2005, vol. 50(11), pp 1785-1789.

[12] T. Floquet, C. Edwards and S. Spurgeon, "on sliding mode observers for systems with unknown inputs", International Journal of Adaptive Control and Signal Processing 2007, vol. 21, pp 638-656.

[13] A. Levant, "Robust exact differentiation via sliding mode technique", Automatica 1998, vol. 43, pp 379-384.

[14] J.A. Moreno, "A Linear Framework for the Robust Stability Analysis of a Generalized Super-Twisting Algorithm", in Proceedings of the 6th IEEE International Conference on Electrical Engineering, Computing Science and Automatic Control (CCE'09), pp 1-6, Toluca, Mexico.

[15] K. Glover and D. McFarlane, "Robust stabilization of normalized coprime factor plant descriptions with $\mathrm{H}_{\infty}$ bounded uncertainty". IEEE Transactions on Automatic Control 1989, vol. 34(8), pp 821830 . 